\title{
De la démocratie en Amérique latine
}

par Javier Santiso, Chef économiste et Directeur adjoint du Centre de développement

- La capacité analytique en matière de réformes économiques est inégale d'un pays à l'autre de l'Amérique latine. Il s'agit là d'un enjeu central pour les démocraties émergentes.

- Cette capacité analytique est cependant une condition nécessaire mais non suffisante pour atteindre une meilleure qualité des réformes économiques : tout aussi centrale est la capacité de mise en œuvre.

- L'articulation entre ces deux capacités, capacité analytique et capacité de mise en œuvre, est sans doute l'un des leviers centraux sur lequel pourrait agir la coopération internationale.

Alexis de Tocqueville n'a pas manqué de le souligner dans ses écrits sur les Amériques : la vitalité de la société civile est capitale pour les démocraties. Cette question est encore plus pressante dans les démocraties émergentes. Elle passe en particulier par un partage du savoir et de la connaissance accrus, auxquels concourent les médias, les agences gouvernementales mais aussi les centres d'analyse (think-tanks) et les universitaires.

En Amérique latine, la présence de ces centres d'analyse, si elle est parfois remarquable, n'en reste pas moins une réalité exsangue. Si dans l'hémisphère nord, les réservoirs de savoirs sur les enjeux et réformes économiques abondent, dans la partie sud ils sont non seulement plus rares mais dotés de moyens plus réduits. Aucun ne dispose, par exemple, d'un fonds de dotation (endowment) c'est-à-dire un capital de départ dont les revenus générés alimentent le budget de fonctionnement annuel, octroyant ainsi une autonomie financière indispensable à l'autonomie intellectuelle. A titre d'exemple, les fonds de dotation de la Brookings ou de I'Institute of International Economics atteignent, en 2005, 220 millions et 150 millions de dollars respectivement. Rien de comparable en Amérique latine, où I'un des plus prestigieux et efficaces centres d'analyse de la région, I'institution colombienne Fedesarrollo, dirigée par Mauricio Cárdenas, peine a boucler un budget annuel de 1.3 millions de dollars, sans la sécurité de bénéficier d'une mise initiale le mettant à l'abri des aléas et déconvenues conjoncturels.
Les centres d'analyse latino-américains ne déméritent pourtant nullement. Historiquement, CIEPLAN par exemple a joué un rôle central dans la transition démocratique chilienne, fournissant pendant des années une ombrelle institutionnelle pour permettre à toute une génération de démocrates de fermenter leurs propositions de réformes gouvernementales. Une fois la démocratie revenue, l'institution a fourni l'essentiel des cadres et ministres chiliens des premiers gouvernements démocratiques, à commencer par son fondateur Alejandro Foxley, aujourd'hui de nouveau au service de l'Etat chilien (il est devenu Ministre des Affaires Etrangères en 2006). Non loin du Chili, une institution comme CERES en Uruguay, dirigée par Ernesto Talvi, se démène pour contribuer aux débats économiques du pays tandis, qu'au Mexique, le CIDE s'est imposé comme un des fleurons des débats démocratiques.

La création d'une communauté épistémique démocratique, capable d'adopter et adapter aux contextes latinoaméricains, des concepts, idées et processus, est d'une importance vitale pour ces jeunes démocraties. Il n'en reste pas moins que cette densité d'institutions cognitives fait souvent défaut. En outre, même si parfois la capacité analytique peut-être singulièrement dense, la capacité de mise en œuvre des réformes peux faire cruellement défaut. L'exemple de l'Argentine atteste s'il le faut combien cette capacité de mise en œuvre efficace doit pouvoir aller de pair avec une capacité cognitive importante. 
L'articulation des deux, capacité d'analyse et capacité de mise en œuvre, est en ce sens au cœur des réussites et échecs réformateurs dans la région. Le paradoxe argentin aura été d'offrir une des plus grandes fêtes réformatrices des années 90 , le pays caracolant en tête de tous les indices de la Banque interaméricaine et de la CEPAL, assise sur une capacité analytique sans pareil dans la région, et un des plus spectaculaire crash économique et financier la décennie suivante.

Non seulement la capacité d'analyse est centrale, mais la capacité de mise en œuvre des réformes l'est tout autant. L'un des enjeux majeurs de l'Amérique latine est non seulement de consolider cette capacité d'analyse endogène, que ce soit au niveau des centres d'analyse et agences gouvernementales qu'au niveau de la capacité de recherche de la société civile, mais aussi de mettre en œuvre des rouages et des courroies de transmissions d'implémentation des réformes. L'émergence de technopoles démocratiques va ainsi de pair avec une participation active aux maillages d'argumentations et contre-argumentations dans la sphère publique. II appelle surtout un processus d'appropriation, d'adaptation et d'adoption auquel se doivent de participer aussi bien les experts que les citoyens. Enfin, il invite à se pencher sur une des lacunes récurrentes des processus de formulation des politiques, à savoir la finalisation, la mise en œuvre des réformes, économiquement, socialement et politiquement acceptables.
Dans la région, trop souvent la politique des experts a donné lieu, comme on l'a montré dans un livre récemment publié*, à des modèles armés et désarmés sur des réalités sociales sans processus d'adoption ni adaptation. Les expérimentations, qu'elles aient été structuralistes ou monétaristes, marxistes ou néo-libérales, ont ainsi abouti à des impasses. Comme le montre une fois encore la trajectoire chilienne, la lente et récurrente fermentation des idées, leur distillation dans les débats d'experts et de citoyens, puis le passage à l'acte, sont autant de maillons essentiels d'une chaîne indispensable pour mettre en action les poulies du développement économique.

\section{Pour approfondir la question :}

* Santiso, J. (2005), Amérique latine : révolutionnaire, libérale, pragmatique, Autrement, Paris.

"Ulysses, the Sirens and the Art of Navigation: Political and Technical Rationality in Latin America", par Javier Santiso et Laurence Whitehead, OECD Development Centre Working Paper No. 256, septembre 2006, Paris. 